\title{
BULÍMICAS:
}

\section{ELAS VÃO À ACADEMIA DE GINÁSTICA?}

\author{
MS. ANDRÉIA WEIS \\ Programa de Pós-graduação em Educação Física, Universidade \\ Federal do Paraná (Curitiba - Paraná - Brasil) \\ E-mail: andreia_weis@hotmail.com
}

\section{MS. PAULO HENRIQUE SANTOS DA FONSECA \\ Instituto Federal de Educação, Ciência e Tecnologia de Santa Catarina \\ (Campus Itajaí) (Itajaí - Santa Catarina - Brasil) \\ E-mail: paulo.fonseca@ifsc.edu.br}

\author{
DR. MARCELO DA SILVA VILLAS BÔAS \\ Departamento de Educação Física, Universidade \\ Estadual de Maringá (Maringá - Paraná - Brasil) \\ E-mail:marcelovb@bol.com.br
}

\author{
DRA. JOICE MARA FACCO STEFANELLO \\ Departamento de Educação Física, Universidade \\ Federal do Paraná (Curitiba - Paraná - Brasil) \\ E-mail: joice@ufpr.br
}

\author{
DR. RICARDO WEIGERT COELHO \\ Departamento de Educação Física, Universidade \\ Federal do Paraná (Curitiba - Paraná - Brasil) \\ E-mail: coelhoricardo@ufpr.br
}

\section{RESUMO}

O objetivo da pesquisa foi determinar a prevalência de mulheres com sintomas de bulimia nervosa frequentadoras de academia de ginástica e verificar, dentre as praticantes que apresentam traços bulímicos, qual a frequência de utilização do exercício físico como forma compensatória. Participaram do estudo 197 mulheres matriculadas em academias de ginástica da cidade de Marechal Cândido Rondon - PR (28,7 99,9 anos). Para avaliar o quadro de bulimia utilizou-se o Teste de Investigação Bulímica de Edingurgh (BITE) e uma anamnese estruturada pelos pesquisadores para atender interesses do estudo. Na análise dos dados foi utilizada a estatística descritiva e a frequência amostral pelo programa estatístico SPSS for Windows 1 I.5. A prevalência de sintomas de bulimia foi de 3\%. A prevalência de traços bulímicos foi de II,2\%. Dos indivíduos que apresentaram traços bulímicos, 59, I\% afirmaram 
praticar exercício físico após episódios de alimentação exagerada. Estes resultados podem indicar que esse comportamento compensatório através da prática de exercício físico pode levar as mulheres a procurar as academias de ginástica.

PALAVRAS CHAVE: Bulimia; exercício fisico; academias de ginástica; transtorno alimentar.

\section{INTRODUÇÃO}

A preocupação com o culto ao corpo atinge as mais diferentes classes sociais, faixas etárias e setores da sociedade em geral. A necessidade de se enquadrar em padrões faz com que sejam lançados esforços e tentativas insanas, que podem resultar em consequências gravíssimas. Algumas destas consequências estão relacionadas ao aparecimento dos quadros de transtornos alimentares, principalmente a anorexia nervosa e bulimia nervosa, desordens que afetam o psíquico alterando a percepção corporal (FONSECA; RENA, 2008). Apesar de muitos acreditarem como sendo quadros clínicos atuais, os transtornos alimentares afetam indivíduos há várias gerações (CORDÁs; CLAUDINO, 2002).

Estes transtornos acometem principalmente adolescentes e mulheres jovens em idade reprodutiva, mas, em proporções menores (representando apenas I0\% dos casos) também são identificadas em homens (MAGALHÃES; MENDONÇA, 2005). Têm origem multifatorial, podendo ser desencadeadas por fatores biológicos, genéticos, psicológicos, socioculturais e familiares (MAGALHÃES; MENDONÇA, 2008, BORGES et al., 2006 ).

Os transtornos alimentares caracterizam-se desde a total abdicação de comida à extrema compulsão alimentar. A privação alimentar, seguida por episódios alimentares compulsivos, acarretam sentimentos de culpa, pela grande quantidade de comida ingerida, que necessitam ser aliviados através de métodos compensatórios. Esse método, identificado como bulimia nervosa (WHO, 1992; APA, 1004), é considerado como um transtorno alimentar específico desde 1980, com a publicação do DSM-III.

Os comportamentos compensatórios dos indivíduos com bulimia nervosa visam "eliminar" os alimentos ingeridos, e podem ser caracterizados como purgativos, com vômitos autoinduzidos, abuso de diuréticos e laxantes e não purgativos, como o jejum e a prática de exercício físico (APA, 1994).

O exercício físico tem se constituído como uma ferramenta comumente utilizada para perda e manutenção de peso pelo indivíduo com bulimia nervosa, pois estes são mais suscetíveis a iniciarem a prática de exercício físico motivados pelo efeito compensatório que esse pode exercer sobre o seu organismo (STRIGELMOORE; ROSSELI et al., 2009). Porém, pessoas com bulimia realizam sessões de 
treinamento que se estendem por horas e que são executadas de forma exacerbada e, por consequência, o exercício passa a ser uma arma contra o seu próprio corpo, acarretando em problemas de ordem física e social.

Alguns estudos (SUNDGOT-BORGEN, 1993; ASSUNÇÃO; CORDÁS et al., 2002; BOSI; OLIVEIRA, 2004) já haviam detectado a relação entre a bulimia e a prática de exercício físico. Porém, não houve estudos que buscassem analisar a prevalência de bulímicos em ambientes de prática de exercício físico e que não fossem atletas.

Assim, diante da crescente proliferação dos ambientes para a prática de exercício físico em academias de ginástica, muitas vezes em decorrência da forte ênfase dada pela mídia acerca destes estabelecimentos como o ambiente adequado para a prática de exercício físico, procurou-se no presente estudo determinar a prevalência de mulheres frequentadoras de academia de ginástica que apresentam bulimia nervosa e verificar com que frequência essas mulheres utilizam o exercício físico como forma compensatória para esse tipo de transtorno alimentar.

\section{PROCEDIMENTOS METODOLÓGICOS}

O presente estudo caracterizou-se como uma pesquisa epidemiológica descritiva, através de dados primários, examinando a prevalência de bulimia nervosa em mulheres que se exercitam em academias de ginástica.

Compõe a população do estudo mulheres praticantes de exercício físico das 10 academias de ginástica existentes na cidade de Marechal Cândido Rondon - PR. A amostra foi calculada utilizando o procedimento sugerido por Barbetta (2008): sabendo-se que o tamanho da população de mulheres que praticavam exercício físico em academias na cidade era de 382, e aceitando um erro amostral tolerável de 5\%, a amostra foi determinada em 195 participantes; no entanto, foram coletados dados de 197 indivíduos, os quais selecionados de forma aleatória e posteriormente convidados a participar do estudo.

A amostra apresentou tempo de prática de exercício físico na academia superior a um mês para aproximadamente $75 \%$, a média da sessão de treinamento em até Ih e meia foi de 83,8\%, um pouco mais da metade da amostra (52,3\%) é solteira, sem filhos (66\%), com faixa de renda mensal entre 3 e 10 salários mínimos (60,4\%), e $84,4 \%$ das mulheres tem no mínimo o ensino médio completo.

Os dados foram coletados nos meses de agosto e setembro do ano de 2009. Através de consentimento para efetuar a pesquisa, foi assinado o termo de autorização da academia e a aplicação dos questionários nas avaliadas seguiu da seguinte forma: as alunas que chegavam ou já se encontravam na academia recebiam 
o convite para participar da pesquisa, momento em que era explicada a intenção da pesquisa e, com o seu aceite, Ihe era entregue o termo de consentimento livre e esclarecido, posteriormente era realizada a aplicação do questionário.

Para avaliar a presença do comportamento bulímico, utilizou-se o questionário autoaplicável Bulimic Inventory Teste Edinburgh (BITE), traduzido para português por Cordás \& Hochgraf ( 1993), o qual teve o seu nível de confiabilidade medido por Magalhães e Mendonça (2005). A utilização do BITE para avaliar bulimia nervosa em praticantes de exercício físico já foi realizada em outros estudos (BOSI; OLIVEIRA, 2004; RIBEIRO, 1995).

O BITE avalia predominantemente comportamentos bulímicos, como ingestão excessiva de alimentos e os métodos purgativos utilizados para compensar estes episódios exagerados de alimentação e que levam o indivíduo a sentir-se mal, tais como indução de vômitos, realização de jejum, uso de laxantes, diuréticos, anorexígenos e dieta.

instrumento fornece resultados em duas escalas: uma de gravidade, e outra de sintomas. Neste estudo será analisado somente a escala de sintomas, que constitui-se de 30 questões, variando de 0 até 30 pontos. A pontuação na escala sintomática pode ser subdividida em quatro grupos: de 20 ou mais pontos (com sintomas de bulimia); pontuação de 15 - 19 (grupo subclínico), de 10 - 14 (hábito alimentar não usual) e pontuação abaixo de 10 (sem sintomas de bulimia).

No presente estudo adotou-se como ponto de corte o valor I 5 na escala sintomática, considerando os valores menores que 15 como sem traços de bulimia nervosa e os valores superiores a esse ponto como contendo traços do transtorno. Este ponto de corte para detecção do transtorno também foi adotado por Cenci et al., (2009), pois, mesmo não alcançando uma pontuação $\geq 20$, os indivíduos que apresentam acima de 15 pontos podem refletir um grupo subclínico de bulímicos, seja na fase inicial do transtorno ou no final do tratamento, exigindo a mesma atenção dos quadros confirmados.

Juntamente com o BITE foi acrescida a pergunta "Se você respondeu sim à questão 24, logo após este episódio de alimentação exagerada você praticou exercício físico como forma de se sentir melhor? Sim ( ) Não ( )". Este questionamento teve a finalidade de responder ao segundo objetivo do estudo, que se propôs a analisar com que frequência mulheres com bulimia nervosa utilizam o exercício físico como forma compensatória.

Utilizou-se a estatística descritiva e de frequência amostral ilustrada por meio de gráficos em forma de pizza. Os cálculos estatísticos e os gráficos foram executados pelo programa estatístico Statistical Package for the Social Sciences for Windows (SPSS I I.5). 


\section{RESULTADOS}

A Figura I caracteriza a frequência em que as quatro escalas sintomáticas do BITE aparecem. Percebe-se que 64,5\% (I 27 sujeitos) não apresentam sintomas de bulimia nervosa, 23,9\% (47 sujeitos) foram classificados como tendo hábito alimentar não usual, 8,6\% ( 17 sujeitos) caracterizaram-se como grupo subclínico e $3 \%$ (6 sujeitos) apresentaram sintomas de bulimia nervosa.

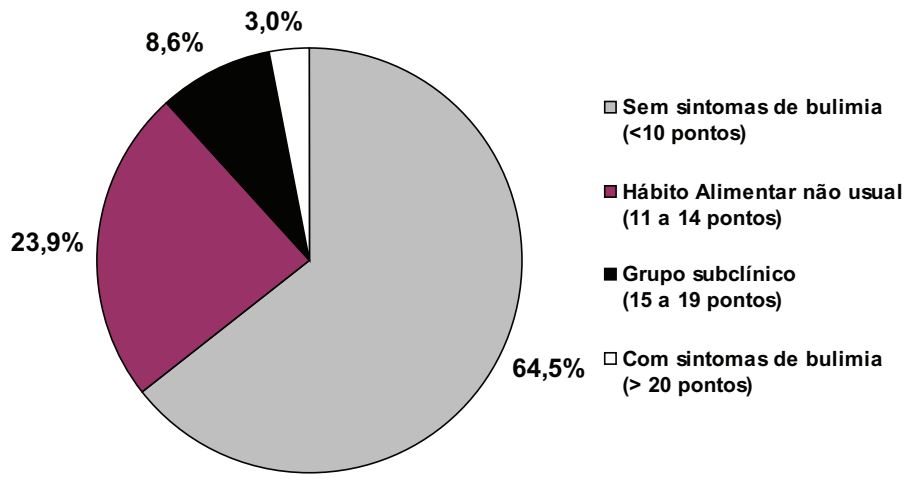

Figura I. Frequência das escalas sintomáticas determinadas pelo BITE $(n=197)$

Na Figura 2 estão apresentados os valores de prevalência de bulimia nervosa, utilizando o ponto de corte adotado neste estudo. Constata-se que $88,8 \%$ ( I 75 sujeitos) não apresentam traços bulímicos e que I I,2\% (22 sujeitos) foram diagnosticados como tendo traços bulímicos.

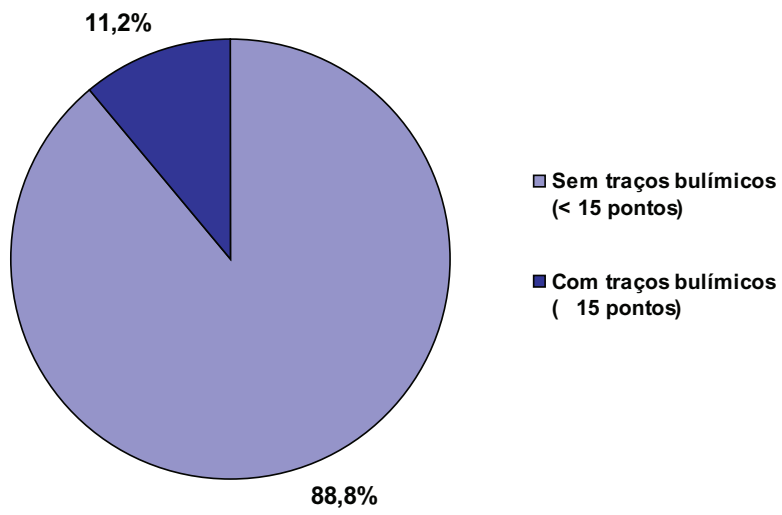

Figura 2. Prevalência de bulimia nervosa entre as praticantes de exercício físico em academias ( $n=197)$. 
Dentre as mulheres que foram diagnosticadas com traços de bulimia nervosa, 40,9\% (9 sujeitos) não praticam exercícios após episódios de compulsão alimentar. Em contrapartida, 59, I \% ( 13 sujeitos) utilizam o exercício físico como forma compensatória após os episódios de alimentação exagerada.

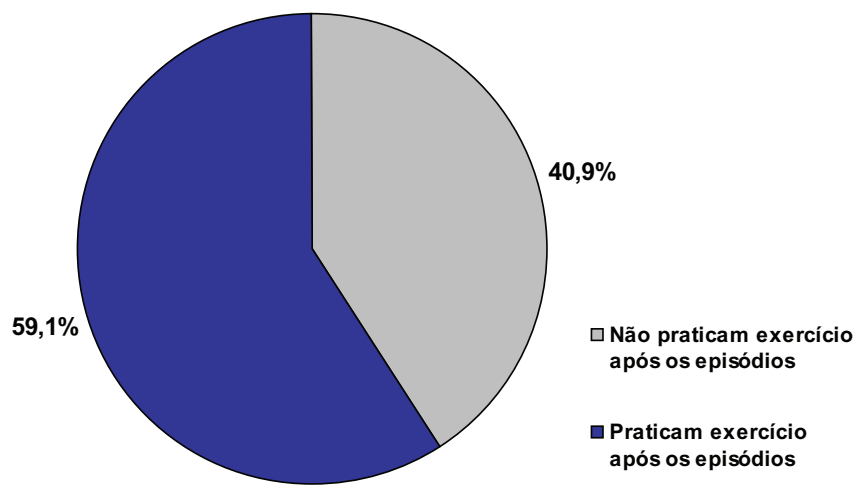

Figura 3. Frequência de utilização do exercício físico como forma compensatória após episódios de alimentação exagerada das praticantes com diagnóstico bulímico ( $n=197)$.

\section{DISCUSSÃO}

Indivíduos que apresentam tendência a desenvolver bulimia nervosa podem usar o exercício inapropriadamente, desta forma há alta probabilidade dessas pessoas entrarem em contato com o profissional da educação física, especialmente no ambiente de academia de ginástica. Essa afirmação se comprova com os resultados apresentados no presente artigo, onde a prevalência de sintomas de bulimia nervosa na amostra foi de 3\% (6 sujeitos). O valor encontrado corrobora com a afirmação de Hoek e Van Hoeken (2003), de que a bulimia é encontrada em até 4\% das mulheres jovens, e demais autores (BORGES et al., 2006; CENCl et al., 2009; TEIXEIRA et al., 2009), na qual indicam o transtorno como o quadro mais prevalente entre os transtornos alimentares na população em geral.

No entanto, como o ponto de corte adotado neste estudo foi de I 5 pontos no BITE, os próximos dados serão discutidos considerando a união dos grupos com sintomas de bulimia nervosa e grupo subclínico, identificados como tendo traços bulímicos.

Neste contexto, Cenci et al., (2009) identificaram em uma amostra de estudantes universitárias, a prevalência de 3,6\% de sintomas de bulimia, II,8\% de 
sintomas subclínicos e 20,9\% de comportamentos alimentares não usuais, valores semelhantes aos achados neste trabalho. Realizando a análise de presença de traços bulímicos apresentada na Figura 2, percebe-se que 88,8\% (I 75 sujeitos) não apresentam traços bulímicos, e I I,2\% (22 sujeitos) foram diagnosticados como tendo traços bulímicos. Este mesmo ponto de corte foi adotado no estudo de Cenci et al., (2009), onde os valores encontrados para esta classificação foram de 84,6\% para ausência de traços bulímicos e 15,4\% para a presença de traços bulímicos, reforçando os valores apresentados anteriormente.

Os valores para traços bulímicos $(11,2 \%)$ se assemelham aos achados de Thomas et al., (2005). A amostra desse estudo foi composta por 239 bailarinas afiliadas às companhias de ballet dos Estados Unidos. Para representantes de companhias locais a prevalência foi de 13\%, em dançarinas de escolas regionais foi de 11,9\% e, para as de nível nacional, os valores alcançaram 13,7\%. Nesta comparação deve ser considerada a influência do tipo de instrumento utilizado em cada estudo, Thomas et al., (2005) utilizaram o Eating Disorder Inventory (EDI). Além das questões inerentes de cada instrumento, as avaliadas foram questionadas sobre dieta, jejum, compulsão alimentar, autoindução vômitos, uso de laxantes, ou exercício fora da aula de ballet.

Em relação à frequência de utilização do exercício físico como forma compensatória. Assunção et al. (2002) avaliaram 47 pacientes ( 3 com anorexia nervosa, 32 com bulimia nervosa e 2 com transtorno alimentar não especificado) com o objetivo de analisar os métodos utilizados para perda de peso. No total da amostra, $70 \%$ relataram o exercício físico como um dos métodos usados para controle ou perda de peso.

Davis (1997), por sua vez, confirma que 55\% dos pacientes com bulimia nervosa praticam exercício físico de forma compulsiva em algum momento de sua história clínica. Tais afirmações corroboram com os achados do presente estudo, demonstrando um valor ainda maior (59,1\% - 13 sujeitos) das mulheres que apresentam traços bulímicos e que praticam exercícios físicos após episódios de alimentação exagerada.

Dixe (2007), em uma amostra de ambos os sexos, verificou que, entre I4 e 25 anos, até 53,6\% dos indivíduos praticavam exercício físico como forma de influenciar o peso, sendo a forma compensatória mais utilizada.

A relação do exercício, comportamento alimentar e qualidade de vida em 3.472 mulheres, com idade entre 18 e 42 anos, que praticavam exercício regularmente foi verificada por Mond et al., (2006). Os autores afirmaram que 37, I \% dos indivíduos com episódios bulímicos praticavam exercício compulsivo.

Crowther et al., (2008), utilizando os instrumentos Eating/Dieting Questionnaire (EDQ), Bulimia Test (BULIT) e Bulimia Test-Revised (BULIT-R) verificaram em 
um estudo longitudinal de 1990 a 2004, que a média entre todos os anos, em uma amostra de 6.844 mulheres, foi de 18,2\% para prática de exercício físico de forma excessiva entre as bulímicas. Este valor é muito inferior ao apontado pela literatura e pelos achados no presente estudo para mulheres com diagnóstico de bulimia nervosa. Striegel-Moore et al., (2009) em um estudo com 3.7। 4 mulheres entre I 8 e 35 anos, buscaram analisar se elas haviam se engajado em algum comportamento compensatório para perda de peso, após o momento de orgia alimentar. Os autores encontraram como resultado que $10 \%$ das mulheres haviam tido na semana anterior ao questionamento alguma orgia alimentar e $6 \%$ tinham utilizado o exercício físico como comportamento compensatório, sendo esse comportamento superior ao vômito (3,7\%) e uso de laxativos (3, l \%), e ficando atrás somente do jejum (6,3\%).

$\bigcirc$ presente estudo pode ser limitado pelo instrumento que foi utilizado, pois o diagnóstico da bulimia foi feito apenas pela aplicação de um questionário. A característica de ser autopreenchível é uma limitação para que seja estabelecido um diagnóstico preciso de bulimia nervosa, já que a interpretação de cada pergunta depende do próprio indivíduo (CENCl et al., 2009). Além disso, os questionários foram aplicados em grupo, o que pode ser outra limitação. Porém, a aplicação de questionários tem sido um consenso em estudos epidemiológicos para a avaliação da bulimia nervosa (BOSI; OLIVEIRA, 2004; STRIEGEL-MOORE; ROSSELI et al., 2009; RIBEIRO, 1995; CENCI, PERES et al., 2009; MOND et al., 2006; CROWTHER; ARMEY et al., 2008). São recomendáveis para a utilização pela facilidade de administração, eficiência e economia, podendo ser aplicados em grandes populações, permitindo aos respondentes revelar um comportamento que poderia deixá-los constrangidos em uma entrevista clínica (FREITAS; GORENSTEIN et al., 2002).

Os resultados obtidos neste estudo, somados à análise dos resultados das pesquisas relatadas anteriormente, sugerem que o exercício físico para mulheres que sofrem de bulimia pode estar sendo praticado com a finalidade equivocada e por consequência dos efeitos dos transtornos. Estes dados podem indicar que esse comportamento compensatório através da prática de exercício físico pode levar as mulheres a procurarem as academias de ginástica. Nesta situação, as mulheres que sofrem de bulimia encontram nas academias de ginásticas uma variedade de práticas corporais para saciarem e reforçarem o comportamento bulímico.

Assim, surge a necessidade dos profissionais de Educação Física serem alertados quanto à existência dessas doenças, como também, estarem preparados para oferecer assistência aos alunos quando parecer apropriado. $\bigcirc$ educador físico pode oferecer conselhos educativos ao cliente, monitorar o programa de exercício físico, como também orientar sobre atitudes prejudiciais à saúde de seu aluno. Deste modo, cabe ao educador físico, em conjunto aos demais profissionais de saúde, estarem atentos e preparados para lidar com os transtornos alimentares e seus agravos, ou ainda, com outros tipos transtornos. 
Com base nos resultados obtidos, observou-se que a prevalência de traços de bulimia nervosa em mulheres frequentadoras de academias de ginástica na cidade de Marechal Cândido Rondon - PR alcançou um valor consideravelmente alto e que merece atenção dos profissionais que trabalham com essas mulheres.

O estudo revelou que a população estudada representa um grupo sujeito à instalação da bulimia nervosa, uma vez que os traços bulímicos encontraram-se presentes na amostra analisada.

Foi identificado também que, das mulheres que contem traços bulímicos, algumas afirmam praticar exercício físico após episódios de alimentação exagerada. Desta forma, confirmando o exercício físico como um método de compensar a falta de controle com a comida.

Através dos resultados encontrados no presente estudo, reforça-se a afirmação de que o profissional de educação física auxilie na identificação de possíveis bulímicos dentro das academias de ginástica, para que este seja educado em relação aos verdadeiros benefícios que o exercício físico pode lhe proporcionar ou para que possa encaminhá-lo à ajuda especializada.

\section{Bulimics: Do They Go to the Gym?}

ABSTRACT: The research objective was to determine the prevalence of women with symptoms of bulimia nervosa attending the gym and check out the practitioners who show bulimic traces, and how often they use the exercise as a compensatory way. The study included 197 women enrolled in gyms in the city of Marechal Cândido Rondon - PR (28.7 \pm 9.9 years). To evaluate the framework of bulimia it was used the Bulimic Investigatory Test of Edingurgh (BITE) and a structured interview by the researchers to meet the interests of the study. For the data analysis it was used descriptive statistics and frequency sampling by the statistical program SPSS for Windows 11.5. The prevalence of symptoms of bulimia was 3\%. The prevalence of bulimic traits was $11.2 \%$. From the individuals that show bulimic traits, 59.1\% reported physical exercise after episodes of over-eating. These results may indicate that this compensatory behavior through physical exercise can cause women to seek out gyms.

KEYWORDS: Bulimia; exercise; fitness centers; eating disorder.

\section{Bulímicos: van al gimnasio?}

RESUMEN: El objetivo de la investigación fue determinar la prevalencia de mujeres que frecuentan al gimnasio y presentan síntomas de la bulimia nerviosa, observar en los practicantes que presentan rasgos de bulimia, con qué frecuencia se utilizan del ejercicio como una forma de compensación. El estudio incluyó a 197 mujeres que participaron en los gimnasios en la 
ciudad de Marechal Cândido Rondon - PR (28,7 \pm 9,9 años). Para evaluar el marco de la bulimia se utilizó la Prueba Investigadora de la Bulimia de Edingurgh (BITE) y una entrevista estructurada por los investigadores para satisfacer a los intereses del estudio. En el análisis de los datos se utilizó estadística descriptiva y la frecuencia de muestra por el programa estadístico SPSS / I.5 for Windows. La prevalencia de síntomas de la bulimia fue de 3\%. La prevalencia de rasgos bulímicos fue del I 1,2\%. De las personas que tienen rasgos bulímicos, el 59, I \% reportó el ejercicio físico después de los episodios de comer en exceso. Estos resultados indican que este comportamiento compensatorio a través del ejercicio físico hace con que las mujeres busquen a los gimnasios.

PALABRAS CLAVE: Bulimia; ejercicio; centros de gimnasia; trastornos de la alimentación.

\section{REFERÊNCIAS}

AMERICAN PSYCHIATRIC ASSOCIATION (APA). Diagnostic and statistical manual of mental disorders. 4th ed. rev. Washington: APA, 1994.

ASSUNÇÃO, S. S.; CORDÁS, T. A.; ARAÚJO L. A. S. B. Atividade física e transtornos alimentares. Revista de Psiquiatria Clínica, São Paulo, v. 29, n. I, p. 4-13, 2002.

BARBETTA, P. A. Estatística aplicada às ciências sociais. Florianópolis: Ed. da UFSC, 2008.

BORGES, N. J. B. et al. Transtornos alimentares: quadro clínico. Medicina, Ribeirão Preto, v. 39, p. 340-348, 2006.

BOSI, M. L. M.; OLIVEIRA, F. P. Comportamentos bulímicos em atletas adolescentes corredoras de fundo. Revista Brasileira de Psiquiatria, São Paulo, v. 26, n. I, p. 32-34, 2004.

CENCI, M. et al. Prevalência de comportamento bulímico e fatores associados em universitárias. Revista de Psiquiatria Clínica, São Paulo, v. 36, n. 3, p. 83-88, 2009.

CORDÁS, T. A.; CLAUDINO, A. M. Transtornos alimentares: fundamentos históricos. Revista Brasileira de Psiquiatria, São Paulo, v. 24, supl. 3, p. 3-6, 2002.

CORDÁS, T. A.; HOCHGRAF, P. B. O bite: instrumento para avaliação da bulimia nervosa. Jornal Brasileiro de Psiquiatria, Rio de Janeiro, v. 2, n. 3, p. |4|-|44, 1993.

CROWTHER, J. H. et al. The point prevalence of bulimic disorders from 1990 to 2004. International Journal of Eating Disorders, Los Angeles, v. 4I, n. 6, p. 49I-497, 2008.

DAVIS, C. Eating disorders and hyperactivity: a psychobiological perspective. Canadian Journal of Psychology, Quebec, v. 42, n. 2, p. 168-75, 1997.

DIXE, M. A. Prevalência das doenças do comportamento alimentar. Análise Psicológica, Lisboa, v. 25, n. 4, p. 559-569, 2007.

FONSECA, S. L.; RENA, L. C. C. B. Transtornos alimentares na adolescência: em busca do corpo ideal. Mosaico: estudos em psicologia. Belo Horizonte, v. 2, n. I, p. 9- I5, 2008. 
FREITAS, S.; GORENSTEIN, C.; APPOLINARIO, J. C. Instrumentos para a avaliação dos transtornos alimentares. Revista Brasileira de Psiquiatria, São Paulo, v. 24, supl. 3, p. 34-38, 2002.

HOEK, H.; VAN HOEKEN, D. Review of the prevalence and incidence of eating disorders. International Journal of Eating Disorders, Los Angeles, v. 34, n. 4, p. 383-396, 2003.

MAGALHÃES, V. C.; MENDONÇA, G. A. S. Transtornos alimentares em universitárias: estudo de confiabilidade da versão brasileira de questionários autopreenchíveis. Revista Brasileira de Epidemiologia, São Paulo, v. 8, n. 3, p. 236-245, 2005.

MOND, J. M. et al. An update on the definition of "excessive exercise" in eating disorders research. International Journal of Eating Disorders, Los Angeles, v. 39, n. 2, p. I 47- I 53, 2006.

RIBEIRO, B. G. Avaliação nutricional de ginastas competitivas de ginástica olímpica. 1995. Dissertação (Mestrado) - Universidade Federal do Rio de Janeiro, Rio de Janeiro, 1995.

STRIEGEL-MOORE, R. H. et al. Gender difference in the prevalence of eating disorder symptoms. International Journal of Eating Disorders, Los Angeles, v. 42, n. 5, p. 47I-474, 2009.

SUNDGOT-BORGEN, J. Prevalence of eating disorders in elite female athletes. International Journal of Sport Nutrition, London, v. 3, n. I, p. 29-33, 1993.

TEIXEIRA, P. C. et al. A prática de exercícios físicos em pacientes com transtornos alimentares. Revista de Psiquiatria Clínica, São Paulo, v. 36, n. 4, p. I 45- I 52, 2009.

THOMAS, J. J.; KEEL, P. K.; HEATHERTON, T. F. Disordered eating attitudes and behaviors in ballet students: examination of environmental and individual risk factors. International Journal of Eating Disorders, Los Angeles, v. 38, n. 3, p. 263-268, 2005.

WORLD HEALTH ORGANIZATION (WHO). The CID-10 classification of mental and behavioral disorders clinical descriptions and diagnostic guidelines. Washington, 1992.

Recebido em: 27 jun. 2011

Aprovado em: 29 dez. 201 I

Endereço para correspondência:

Andréia Weis

Rua Carola Coelho, n¹94, apto I0I,

Bairro Praia Brava

Itajaí - SC

CEP - 88306-828. 